\title{
A numerical method for NNLO calculations
}

\author{
Gudrun Heinrich ${ }^{\mathrm{a} *}$

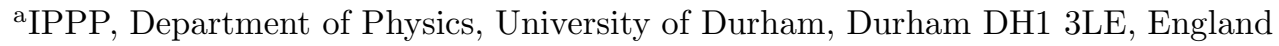

A method to isolate the poles of dimensionally regulated multi-loop integrals and to calculate the pole coefficients numerically is extended to be applicable to phase space integrals as well.

\section{Introduction}

In the last decades, the interplay between increasing precision from the experimental side and NLO predictions becoming available from the theoretical side has led to impressive tests of the Standard Model. However, certain processes or observables in QCD should be known at NNLO in perturbation theory in order to match the experimental precision, and in the domain of electroweak interactions, many NLO processes still await for being calculated exactly.

The calculation of radiative corrections for electroweak processes is mainly complicated by the presence of several mass scales, whereas in QCD the challenge comes from the presence of infrared singularities due to massless partons. If we have to deal with a combination of strong and electroweak interactions, i.e. with singularities and multi-scale problems, the situation is even more involved, and the complexity of the corresponding analytic calculations is enormous. On the other hand, the performance of computers is improving continuously, and in order to calculate cross sections one generally has to use numerical integration at some point in any case. All this points to the fact that a numerical evaluation of the ingredients needed to calculate cross sections beyond leading order, like loop integrals and certain phase space integrals, will be of increasing importance in the future.

In this article I will first present a method which has been developed in [1] to evaluate IR (or UV) divergent multi-loop integrals by extracting the poles in $1 / \epsilon$ and calculating the pole coeffi-

\footnotetext{
*Talk presented at RADCOR/Loops \& Legs 2002, Kloster
} Banz, Germany, September 2002. cients numerically. Then I will outline an extension of this method which makes it applicable to the calculation of phase space integrals as well. Examples from $e^{+} e^{-} \rightarrow 2$ jets will illustrate the procedure.

\section{The method}

The method, called "sector decomposition" [2], is a systematic way to disentangle overlapping divergent regions in parameter space. The algorithm which has been developed to automate this procedure consists of four basic building blocks which will be outlined in the following.

Consider a scalar graph $G$ with $N$ propagators and $L D$-dimensional loop momenta, typically a master integral. The propagators can have powers $\nu_{j}>1$ and not necessarily integer. After Feynman parametrisation, the graph can be written as

$$
\begin{aligned}
G= & \frac{\Gamma\left(N_{\nu}\right)}{\prod_{j=1}^{N} \Gamma\left(\nu_{j}\right)} \int \prod_{j=1}^{N} d x_{j} x_{j}^{\nu_{j}-1} \delta\left(1-\sum_{j=1}^{N} x_{j}\right) \\
& d \mathcal{K}_{1} \ldots d \mathcal{K}_{L}\left[\sum_{j, l=1}^{L} k_{j} M_{j l} k_{l}-2 \sum_{j=1}^{L} k_{j} Q_{j}+J\right]^{-N_{\nu}} \\
= & \frac{(-1)^{N_{\nu}}}{\prod_{j=1}^{N} \Gamma\left(\nu_{j}\right)} \Gamma\left(N_{\nu}-L D / 2\right) \int_{0}^{\infty} \prod_{j=1}^{N} d x_{j} x_{j}^{\nu_{j}-1} \\
& \delta\left(1-\sum_{l=1}^{N} x_{l}\right) \frac{\mathcal{U}^{N_{\nu}-(L+1) D / 2}}{\mathcal{F}^{N_{\nu}-L D / 2}} \\
N_{\nu}= & \sum_{j=1}^{N} \nu_{j}, \quad d \mathcal{K}_{j}=\frac{d^{D} k_{j}}{i \pi^{\frac{D}{2}}}
\end{aligned}
$$




$$
\begin{aligned}
\mathcal{F}(\vec{x}) & =\operatorname{det}(M)\left[\sum_{j, l=1}^{L} Q_{j} \cdot Q_{l} M_{j l}^{-1}-J\right] \\
\mathcal{U}(\vec{x}) & =\operatorname{det}(M) .
\end{aligned}
$$

A necessary condition for the presence of infrared divergences is $\mathcal{F}=0$, whereas $\mathcal{U}=0$ can only lead to an UV divergence. Upon integration over Feynman parameters, both IR and UV poles will manifest themselves as poles in $1 / \epsilon^{a}(a \leq 2 L)$, stemming from endpoint singularities $\left(x_{i}=0\right)$ of the parameters. However, the singular regions are in general overlapping, such that a local subtraction procedure for the poles cannot be immediately applied. Our algorithm iteratively separates the overlapping regions in parameter space by the following steps:

\section{Part I Generation of primary sectors}

The integration domain is split into $N$ "primary sectors" by introducing $\theta$-functions:

$$
\begin{aligned}
\int_{0}^{\infty} d^{N} x & =\sum_{l=1}^{N} \int_{0}^{\infty} d^{N} x \prod_{\substack{j=1 \\
j \neq l}}^{N} \theta\left(x_{l}-x_{j}\right) \theta\left(x_{l}\right) \\
& \Rightarrow G=\sum_{l=1}^{N} G_{l} .
\end{aligned}
$$

Then the $\delta$-distribution in Eq. (1) is eliminated in such a way that the remaining integrations are from 0 to 1 , which can be achieved by the substitution $x_{j}=x_{l} t_{j}$ for $j<l, x_{j}=x_{l}$ for $j=l$ and $x_{j}=x_{l} t_{j-1}$ for $j>l$. Because of general homogeneity properties of $\mathcal{F}$ and $\mathcal{U}, x_{l}$ factorises like

$\mathcal{U}(\vec{x}) \rightarrow \mathcal{U}_{l}(\vec{t}) x_{l}^{L} \quad, \quad \mathcal{F}(\vec{x}) \rightarrow \mathcal{F}_{l}(\vec{t}) x_{l}^{L+1}$

and thus, using $\int \frac{d x_{l}}{x_{l}} \delta\left(1-x_{l}\left(1+\sum_{k=1}^{N-1} t_{k}\right)\right)=1$, one obtains in each primary sector $l$

$G_{l}=\int_{0}^{1} \prod_{j=1}^{N-1} d t_{j} \frac{\mathcal{U}_{l}^{N_{\nu}-(L+1) D / 2}}{\mathcal{F}_{l}^{N_{\nu}-L D / 2}} \quad, \quad l=1, \ldots N$.

Note that the feature that singularities only occur as some Feynman parameters go to zero is preserved by the transformations above.

\section{Part II Iterated sector decomposition}

As after part I the overlapping regions in general are not disentangled yet, i.e. $\mathcal{F}$ or $\mathcal{U}$ still vanish if a certain set of parameters goes to zero, the decomposition into sectors is iterated until $\mathcal{F}_{l}$ and $\mathcal{U}_{l}$ contain a constant term. This iteration produces $k$ new subsectors of a given primary sector $l$, and in each such subsector one has integrals of the form

$G_{l k}=\int_{0}^{1}\left(\prod_{j=1}^{N-1} d t_{j} t_{j}^{A_{j}-B_{j} \epsilon}\right) \frac{\mathcal{U}_{l k}^{N_{\nu}-(L+1) D / 2}}{\mathcal{F}_{l k}^{N_{\nu}-L D / 2}}$,

where now all singularities are factored out in the bracket, while $\mathcal{F}_{l k}$ and $\mathcal{U}_{l k}$ do not lead to singularities anymore.

\section{Part III Extraction of the poles}

$A_{j}<0$ in (3) leads to poles which have to be subtracted. Typical for gauge theories are logarithmic singularities, i.e. $A_{j}=-1$, where the subtractions are of the form

$$
\begin{aligned}
& \int_{0}^{1} d t_{j} t_{j}^{-1+B_{j} \epsilon} \mathcal{I}\left(t_{j}, \epsilon\right)= \\
& \frac{\mathcal{I}\left(t_{j}=0, \epsilon\right)}{B_{j} \epsilon}+\int_{0}^{1} d t_{j} t_{j}^{B_{j} \epsilon} \frac{\mathcal{I}\left(t_{j}, \epsilon\right)-\mathcal{I}(0, \epsilon)}{t_{j}} .
\end{aligned}
$$

For $A_{j}<-1$, the Taylor expansion around $t_{j}=0$ has to be carried out to a higher order, but the procedure works analogously.

After having isolated the poles in this way, the resulting expression can be expanded in $\epsilon$. This leads to a Laurent series for each subsector integral $G_{l k}$, where the expansion in $\epsilon$ can in principle be carried out to arbitrary order $m$ :

$$
G_{l k}=\sum_{j=-m}^{2 L} C_{l k, j} \epsilon^{-j} .
$$

Finally, the $R_{l}$ subsectors and the $N$ primary sectors are summed over to obtain the result for the graph $G$ :

$$
G=\sum_{l=1}^{N} G_{l}=\sum_{l=1}^{N} \sum_{k=1}^{R_{l}} G_{l k} .
$$




\section{Part IV Calculation of the pole coeffi- cients}

What remains to be done is the calculation of the pole coefficients $C_{j}=\sum_{l=1}^{N} \sum_{k=1}^{R_{l}} C_{l k, j}$. These are $(N-1-\tilde{\mathrm{j}})$-dimensional parameter integrals $(\tilde{\mathrm{j}}=\max (\mathrm{j}, 0))$. In principle, one can attempt to perform these integrations analytically, and doing this in an automatized way poses no problem for large values of $j$, i.e. the leading and subleading poles. However, for smaller values of $j$, automatic analytic integration with standard algebraic integration routines is not possible anymore. On the other hand, numerical integration of these functions does not pose any problem as long as the function $\mathcal{F}$ has no singularities ${ }^{2}$ within the integration region. Therefore, for integrals with more than one scale, the numerical points to be calculated are chosen to be in the Euclidean region in order to insure that $\mathcal{F}$ is a regular function. In order to deal with general physical kinematics, more sophisticated numerical integration methods have to be used, as for example the ones suggested in $[3,4]$, which can deal with thresholds in the multileg one-loop case.

\section{Examples for loop integrals}

The method outlined above has been applied to check the results for the planar [5] and nonplanar [6] on-shell massless double box. A prediction [1] for the master integrals of the massless double box with one external leg off-shell has also been made, which has been confirmed by analytical calculations later [7-10]. Massless double boxes with two off-shell external legs also have been calculated. For example, for the planar double box with $p_{3}$ and $p_{4}$ off-shell, one obtains analytically for the leading and subleading poles:

$$
\begin{aligned}
& D B_{m_{3} m_{4}}=\Gamma^{2}(1+\epsilon) \frac{\left(-m_{4}^{2}\right)^{-2 \epsilon}}{s^{2} t}\left\{\frac{1}{4 \epsilon^{4}}-\frac{1}{\epsilon^{3}} \times\right. \\
& {\left[\frac{1}{2} \log \left(s / m_{4}^{2}\right)+\log \left(t / m_{4}^{2}\right)-\frac{1}{2} \log \left(m_{3}^{2} / m_{4}^{2}\right)\right]}
\end{aligned}
$$

\footnotetext{
${ }^{2}$ In contrast to the $1 / \epsilon$ poles which have been extracted already, these are integrable singularities, like for example threshold singularities, but they lead to peaks in multiparameter space which may cause numerical problems.
}

$$
\left.+\mathcal{O}\left(\frac{1}{\epsilon^{2}}\right)\right\} \quad, \quad\left(m_{3,4}^{2}=p_{3,4}^{2}\right) .
$$

Numerically e.g. at the point

$\left(-s,-t,-u,-m_{3}^{2},-m_{4}^{2}\right)=(2 / 3,2 / 3,2 / 3,1,1)$,

the result is

$$
\begin{aligned}
& D B_{m_{3} m_{4}}(2 / 3,2 / 3,2 / 3,1,1)=\Gamma^{2}(1+\epsilon) \\
& \left(-\frac{0.8437}{\epsilon^{4}}-\frac{2.0524}{\epsilon^{3}}-\frac{10.52}{\epsilon^{2}}-\frac{48.62}{\epsilon}-140.77\right) .
\end{aligned}
$$

The accuracy is better than $3 \%$ and can be improved easily at the expense of more integration time.

The most challenging loop integral tackled with this method is the planar 3-loop box. It has been calculated recently by Smirnov [11] who achieved a fully analytical result for the coefficients of $1 / \epsilon^{j}, j=2 \ldots 6$. The numerical result obtained with our method is in agreement with [11].

\section{General multi-parameter integrals}

As the method of sector decomposition is very general and straightforward, it can be applied to integrals other than loop integrals, as for example phase space integrals, as well. However, some properties which are specific to loop integrals, and thus were built into the code for loop integrals, cannot be used anymore in the case of general parameter integrals: $i)$ there is not necessarily a $\delta\left(1-\sum_{l=1}^{N} x_{l}\right)$ constraint as in $(1)$, ii) the universal scaling properties (2) for $\mathcal{F}$ and $\mathcal{U}$ are lost, and - most importantly - iii) the singularities in $\epsilon$ may not only be at $x_{i}=0$ anymore. Taking into account $i$ ) and $i i$ ) is not a big issue, although it lengthens the code substantially. On the other hand, the absence of singularities other than the ones at $x_{i}=0$ is crucial for the program to work. However, endpoint singularities can always be remapped by simple variable transformations such that they occur at $x_{i}=0$ only. As in the case of loop integrals, singularities within the integration region have to be avoided, for instance by transforming the integrand correspondingly before feeding it into the code.

\section{Example from $e^{+} e^{-} \rightarrow 2$ jets at NNLO}

In order to calculate a cross section like $e^{+} e^{-} \rightarrow 2$ or 3 jets at NNLO, one needs the 
following ingredients: The two-loop virtual part, single radiation from one-loop graphs, and double radiation from tree graphs. A lot of progress has been achieved in the past 2 years concerning the first two items. For the case of double radiation where two particles are unresolved, the soft and collinear limits are known, but a systematic procedure to set up a local subtraction scheme which allows to isolate and analytically integrate the infrared singular regions in phase space has not been established yet. Here it is shown that our code is able to isolate the poles and integrate the pole coefficients numerically. The following IR singular limits can be distinguished:

1. three particles collinear

2. two pairs of particles collinear

3. two particles collinear, one soft

4. two particles soft

5. a soft $q \bar{q}$ pair.

As a specific example, we will consider the triple collinear limit. In this limit, phase space and matrix element factorise in the following way:

$$
\begin{aligned}
d \Phi\left(p_{1}, p_{2}, p_{3}, p_{4}\right) & \rightarrow d \Phi^{(2)}\left(\tilde{p}_{123}, \tilde{p}_{4}\right) \times d \Phi^{\mathrm{tc}} \\
|M|^{2} & \rightarrow\left|\tilde{M}_{B}\right|^{2} \times\left\langle P_{g_{1} g_{2} q_{3}}\right\rangle / s_{123}^{2},
\end{aligned}
$$

where $\tilde{p}_{123}$ and $\tilde{p}_{4}$ are defined in analogy to the dipole method [12] for NLO calculations as $\tilde{p}_{123}=p_{1}+p_{2}+p_{3}-\frac{y}{1-y} p_{4}, \tilde{p}_{4}=\frac{1}{1-y} p_{4}, y=$ $s_{\tilde{1} 23} / \tilde{s} \quad\left(\tilde{s}=\left(p_{1}+p_{2}+p_{3}+p_{4}\right)^{2}=\left(\tilde{p}_{123}+\tilde{p}_{4}\right)^{2}\right)$, $\tilde{M}_{B}$ is the 'Born' $1 \rightarrow 2$ matrix element with final state momenta $\tilde{p}_{123}, \tilde{p}_{4}$ and $\left\langle P_{g_{1} g_{2} q_{3}}\right\rangle$ is the spin-averaged triple collinear $q \rightarrow g_{1} g_{2} q_{3}$ splitting function. The triple collinear phase space factor can be cast into the following form $(d=4-2 \epsilon)$

$$
\begin{aligned}
& \int d \Phi^{\mathrm{tc}}=\frac{\tilde{s}^{2-2 \epsilon}}{(4 \pi)^{d}} \frac{1}{\Gamma^{2}(1-\epsilon)} \\
& \int d z_{1} d z_{2} d z_{3} \delta\left(1-\sum_{i=1}^{3} z_{i}\right)\left[z_{1} z_{2} z_{3}\right]^{-\epsilon} \\
& \int_{0}^{1} d y[y(1-y)]^{1-2 \epsilon} \int_{0}^{1} d u[u(1-u)]^{-\epsilon} \\
& \frac{\Gamma(1-\epsilon)}{\sqrt{\pi} \Gamma\left(\frac{1}{2}-\epsilon\right)} \int_{0}^{\pi} d \theta(\sin \theta)^{-2 \epsilon}
\end{aligned}
$$

where $z_{i}=p_{i} \tilde{p}_{4} / \tilde{p}_{123} \tilde{p}_{4} \quad(i=1,2,3)$ and the integral over $u$ is a rescaled transverse momentum integration. In the above parametrisation, the Mandelstam variables are given by:

$$
\begin{aligned}
s_{123} & =\tilde{s} y \\
s_{13} & =\tilde{s} y u\left(1-z_{2}\right) \\
s_{23} & =\tilde{s} y u \frac{z_{1} z_{2}}{\left(1-z_{2}\right)}\left(1+\nu^{2}-2 \nu \cos \theta\right) \\
s_{12} & =\tilde{s} y u \frac{z_{2} z_{3}}{\left(1-z_{2}\right)}\left(1+\lambda^{2}+2 \lambda \cos \theta\right) \\
s_{i 4} & =\tilde{s}(1-y) z_{i} \quad(i=1,2,3) \\
\nu^{2} & =\frac{z_{3}}{z_{1} z_{2}} \frac{(1-u)}{u} \quad, \quad \lambda^{2}=\frac{z_{1}^{2}}{z_{3}^{2}} \nu^{2}
\end{aligned}
$$

The triple collinear splitting functions can be found in $[13,14]$. The aim here is only to demonstrate how the method operates. So let us focus first on the Abelian part of the splitting $q \rightarrow g_{1} g_{2} q_{3}$, described by the splitting function

$$
\begin{aligned}
& \left\langle P_{g_{1} g_{2} q_{3}}^{A b e l}\right\rangle / s_{123}^{2}=C_{F}^{2}\left\{\frac{A(z)}{s_{13} s_{23}}\right. \\
& +\frac{B_{1}(z)}{s_{13} s_{123}}+\frac{B_{2}(z)}{s_{23} s_{123}} \\
& \left.+C\left(\frac{s_{23}}{s_{13} s_{123}^{2}}+\frac{s_{13}}{s_{23} s_{123}^{2}}\right)+\frac{D}{s_{123}^{2}}\right\} .
\end{aligned}
$$

We want to integrate this function over the triple collinear phase space factor given in (4). Using parametrisation (5) for the Mandelstam variables, we see that the $y$-integration factorises immediately, and that the $u$-integration also factorises except if there is an angular dependence through $s_{23}$. In this case, however, one has to insure that no (integrable) singularity in the analytic plane is crossed before feeding the function into the numerical integration routine. This can be achieved easily by doing the $\theta$-integration analytically. Using

$$
\begin{aligned}
& \frac{\Gamma(1-\epsilon)}{\sqrt{\pi} \Gamma\left(\frac{1}{2}-\epsilon\right)} \int_{0}^{\pi} d \theta(\sin \theta)^{-2 \epsilon}\left[1+\nu^{2} \pm 2 \nu \cos \theta\right]^{-1} \\
= & \theta\left(1-\nu^{2}\right)_{2} F_{1}\left(1,1+\epsilon, 1-\epsilon, \nu^{2}\right) \\
+ & \theta\left(\nu^{2}-1\right) \frac{1}{\nu^{2}}{ }_{2} F_{1}\left(1,1+\epsilon, 1-\epsilon, 1 / \nu^{2}\right)
\end{aligned}
$$

one can split the subsequent integrations into two parts at $\nu^{2}=1$. After remapping to integrals from 0 to 1 and using the integral representation 
for the Hypergeometric function, one arrives at the 'standard' form required by the numerical integration routine. The result is given by

$$
\begin{aligned}
I^{\text {Abel }} & =\int d \Phi^{\mathrm{tc}}\left\langle P_{g_{1} g_{2} q_{3}}^{\text {Abel }}\right\rangle / s_{123}^{2} \\
& =\frac{\tilde{s}^{-2 \epsilon}}{(4 \pi)^{d}} \frac{C_{F}^{2}}{\Gamma^{2}(1-\epsilon)} \sum_{i=1}^{4} \frac{P_{i}}{\epsilon^{i}} \\
P_{4} & =4 \pm 2 \times 10^{-6} \\
P_{3} & =13.99999 \pm 4.8 \times 10^{-5} \\
P_{2} & =10.1125 \pm 0.0015 \\
P_{1} & =-38.8109 \pm 0.012 \\
P_{0} & =-116.47 \pm 0.07
\end{aligned}
$$

The same procedure can be followed for the other singular limits of the matrix element.

The most complicated denominator occurs in the non-Abelian part of the triple collinear splitting function, where an angular dependent denominator appears quadratically, $\left\langle P_{g_{1} g_{2} q_{3}}^{\text {non-Ab }}\right\rangle / s_{123}^{2} \sim A^{\prime}(z) / s_{12}^{2}+$ less complicated. However, this does not present a problem for the numerical method. Again, all one has to do is to insure that no singularities in the analytic plane are crossed. Carrying out the angular integration leads to

$$
\begin{aligned}
I_{\theta}= & \frac{\Gamma(1-\epsilon)}{\sqrt{\pi} \Gamma\left(\frac{1}{2}-\epsilon\right)} \int_{0}^{\pi} d \theta(\sin \theta)^{-2 \epsilon} \\
& {\left[1+\lambda^{2}+2 \lambda \cos \theta\right]^{-2}=} \\
& \theta\left(1-\lambda^{2}\right){ }_{2} F_{1}\left(2,2+\epsilon, 1-\epsilon, \lambda^{2}\right) \\
+ & \theta\left(\lambda^{2}-1\right) \frac{1}{\lambda^{4}}{ }_{2} F_{1}\left(2,2+\epsilon, 1-\epsilon, 1 / \lambda^{2}\right)
\end{aligned}
$$

Following the same procedure as outlined for (6) again leads to a representation which can be fed into the numerical routine.

\section{Conclusions}

A constructive algorithm to isolate infrared singularities from multi-loop integrals and phase space integrals has been presented. The algorithm produces finite parameter integrals as pole coefficients, which can be integrated numerically, or analytically in simple cases. For the numerical integration, one has to insure that the function does not have (integrable) singularities within the integration region, which can be achieved easily by appropriate variable transformations in the case of phase space integrals with one overall scale. As applications, a result for a massless double box with two legs off-shell and an example from the phase space of $e^{+} e^{-} \rightarrow 2$ jets have been given. The method can serve to check various kinds of analytical NNLO results numerically and also provides a step towards a completely numerical evaluation of radiative corrections.

\section{Acknowledgements}

I would like to thank V. A. Smirnov for the exchange of results which triggered further developments of the program, and the organisers of the conference for the kind invitation.

\section{REFERENCES}

1. T. Binoth and G. Heinrich, Nucl. Phys. B $\mathbf{5 8 5}$ (2000) 741.

2. K. Hepp, Commun. Math. Phys. 2, 301 (1966); see also contribution of S. Pozzorini in these proceedings.

3. G. Passarino, Nucl. Phys. B 619 (2001) 257; A. Ferroglia, M. Passera, G. Passarino and S. Uccirati, hep-ph/0209219.

4. T. Binoth, G. Heinrich and N. Kauer, hep$\mathrm{ph} / 0210023$.

5. V. A. Smirnov, Phys. Lett. B 460 (1999) 397.

6. J. B. Tausk, Phys. Lett. B 469 (1999) 225.

7. V. A. Smirnov, Phys. Lett. B 491 (2000) 130.

8. T. Gehrmann and E. Remiddi, Nucl. Phys. B 601 (2001) 248.

9. V. A. Smirnov, Phys. Lett. B 500 (2001) 330.

10. T. Gehrmann and E. Remiddi, Nucl. Phys. B 601 (2001) 287

11. V. A. Smirnov, in these proceedings [hepph/020929]; see also Phys. Lett. B 547 (2002) 239.

12. S. Catani and M. H. Seymour, Nucl. Phys. B 485 (1997) 291 [Erratum-ibid. B 510 (1997) $503]$.

13. J. M. Campbell and E. W. Glover, Nucl. Phys. B 527 (1998) 264.

14. S. Catani and M. Grazzini, Phys. Lett. B 446 (1999) 143; S. Catani and M. Grazzini, Nucl. Phys. B 570 (2000) 287. 